\title{
Consumo de "produtos naturais» e «suplementos alimentares» numa Unidade de Saúde Familiar: estudo exploratório
}

Ângela Lee Chin, ${ }^{1}$ Ana Filipa Vicente, ${ }^{2}$ Olena Lourenço ${ }^{3}$

\section{RESUMO}

Introdução: O consumo de «produtos naturais» (PN) e «suplementos alimentares» (SA) tem aumentado de popularidade. Os estudos sobre o consumo destes produtos são importantes, pois existem poucos dados nacionais e a população geralmente desconhece os riscos associados.

Objetivos: Determinar a prevalência do consumo de PN/SA na Unidade de Saúde Familiar (USF) Arruda. Adicionalmente pretendeu-se caracterizar socio-demograficamente os consumidores destes produtos e os seus hábitos de consumos e verificar se existe associação com o género, idade, escolaridade e estado civil.

Tipo de estudo: Observacional, descritivo e transversal.

Local: USF Arruda.

População: Utentes adultos inscritos na USF Arruda.

Métodos: Os dados foram recolhidos numa amostra não aleatória, de conveniência, de 1 de outubro a 30 de novembro de 2016. Foi aplicado um questionário anónimo, confidencial e voluntário. Os dados foram codificados, registados e analisados em SPSS ${ }^{\circledR}$. Resultados: Obtiveram-se 351 questionários válidos. Dos inquiridos, 68,9\% eram mulheres, 72,9\% tinham idade entre 31 e 75 anos, 50,7\% apresentavam escolaridade inferior ou igual a seis anos e 50,9\% eram casados ou viviam em união de facto. $91,2 \%$ referiu consumir ou ter consumido PN/SA nos últimos seis meses. Destes, $64,7 \%$ consumiu um ou dois PN/SA, sendo que $75,9 \%$ fê-lo diariamente. Relativamente à análise da associação entre o consumo de PN/ SA e as variáveis estudadas concluiu-se que apenas não estão associadas para o género.

Conclusões: Os resultados deste estudo apontam para uma elevada prevalência de consumo de PN/SA na USF Arruda. Dada a relevância desta temática e ao facto de existirem poucos estudos atuais nesta área, consideramos que seria pertinente realizar investigações mais robustas, idealmente que pudessem ser representativas do panorama nacional.

Palavras-chave: Produtos naturais; Suplementos alimentares; Médico de família

\section{INTRODUÇÃO}

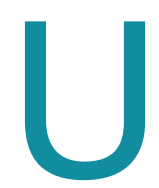

$\mathrm{m}$ «produto natural» $(\mathrm{PN})$ é um composto químico ou substância obtida a partir de plantas, fungos e microrganismos e que normalmente tem atividade biológica ou farmacológica. Tem sido usado no tratamento de várias patologias. ${ }^{1}$

1. UCSP Alverca do Ribatejo, ACeS Estuário do Tejo.

2. USF Arruda, ACeS Estuário do Tejo.

3. UCSP Alenquer, ACeS Estuário do Tejo.
Os «suplementos alimentares» (SA) são géneros alimentícios que, ao constituírem fontes concentradas de determinadas substâncias, nutrientes ou outras, com efeito nutricional ou fisiológico, se destinam a complementar e/ou suplementar o regime alimentar normal. ${ }^{2-5}$

Apesar de a farmacologia de muitos PN/SA ainda não estar bem estudada, a sua utilização tem ganho popularidade. Existem estudos que descrevem efeitos adversos e interações medicamentosas associados ao seu uso, sobretudo nos indivíduos mais velhos e polimedicados. ${ }^{1,6}$ 
Existem várias explicações para o incremento no consumo destes produtos. Uma das possíveis razões deve-se ao facto de o produto ser designado de «natural», fazendo com que o consumidor o associe a segurança. Outras justificações plausíveis são a automedicação, a comercialização destes produtos por laboratórios de renome e os efeitos adversos nos fármacos sintéticos. ${ }^{1}$

Muitas vezes existe um défice na comunicação entre médicos e utentes relativamente ao consumo de PN/SA. Isto poderá ser devido ao facto de os utentes mostrarem algum desconforto em mencionar este consumo, ao seu desconhecimento da sua relevância clínica e por não necessitarem de prescrição médica. Por outro lado, alguns médicos possuem pouco conhecimento relativamente à prevalência do consumo destes produtos, seus potenciais efeitos adversos e interações medicamentosas. ${ }^{6}$

A documentação da União Europeia de apoio à clarificação de enquadramento de um produto como SA ou como medicamento refere que os SA são usados com a finalidade de manter, apoiar ou otimizar os processos fisiológicos normais. Por sua vez, os medicamentos destinam-se a ser administrados quando as funções fisiológicas estão alteradas e também para prevenir doenças. ${ }^{2}$

Relativamente às entidades competentes, a Direção-Geral de Alimentação e Veterinária (DGAV) é a autoridade competente responsável pela notificação aquando da comercialização e da definição das obrigações relativamente a um SA, sendo a Autoridade de Segurança Alimentar e Económica (ASAE) a responsável pela fiscalização do cumprimento das suas normas. A Autoridade Nacional do Medicamento e Produtos de Saúde, I.P. (INFARMED) é a autoridade competente para regular e supervisionar os setores dos medicamentos, dispositivos médicos e produtos cosméticos e de higiene corporal. $^{2}$

No entanto, a maioria dos SA/PN não está sujeita a controlo pelas autoridades competentes na área da saúde (INFARMED), podendo constituir um risco para a saúde pública. ${ }^{1}$

Os estudos sobre os hábitos de consumo destes produtos são extremamente relevantes, uma vez que existem poucos dados em Portugal e a população de um modo geral não tem noção dos riscos associados a uma adesão contínua, indiscriminada e sem acompanhamento por profissionais de saúde. ${ }^{7}$

Neste contexto considera-se pertinente a realização do presente trabalho de investigação.

\section{OBJETIVOS}

Pergunta de investigação:

- Qual a prevalência do consumo de PN/SA nos utentes adultos inscritos na Unidade de Saúde Familiar (USF) Arruda?

Pretende-se:

- Caracterizar socio-demograficamente os consumidores de PN/SA e os seus consumos;

- Verificar se existe associação entre o consumo de PN/SA e o género, a idade, a escolaridade e estado civil.

\section{MÉTODOS}

Realizou-se um estudo observacional, descritivo e transversal, de uma amostra de conveniência da população em estudo (utentes inscritos na USF Arruda, com médico de família e idade acima dos 18 anos) no período entre 1 de outubro e 30 de novembro de 2016.

Admitiram-se como critérios de inclusão: utentes de ambos os sexos, com idade superior a 18 anos e que recorreram à consulta na USF Arruda no período em estudo.

Como critérios de exclusão foram considerados: questionários incorretamente preenchidos, utentes que não aceitassem participar no estudo, doentes com défice cognitivo, grávidas, puérperas e iletrados.

Foram estudadas variáveis qualitativas (género, idade, escolaridade, estado civil, consumo de PN/SA, regularidade de consumo, motivo da toma do PN/SA, informação ao médico assistente) e quantitativas (número de PN/SA).

Foi elaborado um questionário (Anexo 1), o qual foi validado através da aplicação de um pré-teste a sete indivíduos durante o mês de agosto de 2016 para validação do questionário final.

O questionário final foi autopreenchido pelos utentes que recorreram à consulta na USF Arruda no período de estudo.

Para obtenção do tamanho da população em estudo recorreu-se ao MIM@UF®.

Procedeu-se à determinação do número de respos- 
tas ao questionário que seriam necessárias para que a amostra fosse representativa da população em estudo, considerando um intervalo de confiança de $95 \%$.

Os dados obtidos foram registados e analisados utilizando o SPSS $^{\circledast}$.

Este estudo respeitou os princípios da Declaração de Helsínquia modificada em Edimburgo (outubro de 2000), garantindo a confidencialidade e o anonimato acerca da identidade dos participantes, bem como a garantia da utilização dos seus dados apenas para fins estatísticos.

O protocolo do estudo foi entregue e aprovado pela Comissão de Ética para a Saúde da ARSLVT.

\section{RESULTADOS}

O número de utentes a incluir na população em estudo - utentes inscritos na USF Arruda, com médico de família e idade acima dos 18 anos - foi de 7.343 utentes.

No período entre 1 de outubro e 30 de novembro de 2016 foram preenchidos um total de 359 questionários, dos quais foram excluídos oito por se encontrarem incorretamente preenchidos.

Deste modo, obteve-se uma amostra de 351 elementos, sendo que $68,9 \%$ diziam respeito ao género feminino (Quadro I).

Analisando a variável idade (Quadro II) constatou-se que $72,9 \%$ dos elementos encontrava-se na faixa etária compreendida entre os 31 e os 75 anos de idade, sendo que $62,9 \%$ de inquiridos apresentava idade acima de 50 anos.

Verificou-se que cerca de metade dos elementos da amostra $(50,7 \%)$ mencionou ter um grau de escolaridade até ao $6^{\circ}$ ano (Quadro III).

Em relação ao estado civil (Quadro IV) verificou-se que 59,0\% dos inquiridos tinha o estatuto de casado(a)/ / união de facto.

No que concerne à questão Consome ou consumiu nos últimos seis meses algum produto natural/suplemento alimentar? conclui-se que $91,2 \%$ respondeu afirmativamente (Quadro V).

Analisando as respostas à pergunta anterior constata-se que $62,4 \%$ das mulheres responderam afirmativamente. Os grupos etários que apresentaram maior taxa de respostas afirmativas foram os grupos dos 31-50 anos (23,9\%), 51-65 anos (22,2\%) e 65-75 anos $(22,2 \%)$. Relativamente à escolaridade, os que declara-

$\begin{aligned} & \text { QUADRO I. Caracterização demográfica da variável } \\
& \text { «Género» }\end{aligned}$
\begin{tabular}{l|c|l}
\hline Feminino & N & $\%$ \\
\hline Masculino & 109 & 68,9 \\
\hline Total & 351 & 31,1 \\
\hline
\end{tabular}

\begin{tabular}{|c|c|c|}
\hline \multicolumn{3}{|c|}{$\begin{array}{l}\text { QUADRO II. Caracterização demográfica da variável } \\
\text { «Idade» }\end{array}$} \\
\hline & $N$ & $\%$ \\
\hline 18 a 30 anos & 35 & 10,0 \\
\hline 31 a 50 anos & 95 & 27,1 \\
\hline 51 a 65 anos & 83 & 23,6 \\
\hline 65 a 75 anos & 78 & 22,2 \\
\hline Acima de 75 anos & 60 & 17,1 \\
\hline Total & 351 & 100,0 \\
\hline
\end{tabular}

\begin{tabular}{|c|c|c|}
\hline \multicolumn{3}{|c|}{$\begin{array}{l}\text { QUADRO III. Caracterização demográfica da variáve } \\
\text { «Escolaridade» }\end{array}$} \\
\hline & $N$ & $\%$ \\
\hline$<=4$ anos & 114 & 32,5 \\
\hline 5 a 6 anos & 64 & 18,2 \\
\hline 7 a 9 anos & 68 & 19,4 \\
\hline Ensino secundário & 53 & 15,1 \\
\hline Ensino superior & 49 & 14,0 \\
\hline Total & 348 & 99,2 \\
\hline NS/NR & 3 & 0,8 \\
\hline Total & 351 & 100,0 \\
\hline
\end{tabular}

\begin{tabular}{|c|c|c|}
\hline \multicolumn{3}{|c|}{$\begin{array}{l}\text { QUADRO IV. Caracterização demográfica da variável } \\
\text { «Estado civil» }\end{array}$} \\
\hline & $N$ & $\%$ \\
\hline Solteiro(a) & 47 & 13,4 \\
\hline Casado(a)/União de Facto & 207 & 59,0 \\
\hline Divorciado(a) & 27 & 7,7 \\
\hline Viúvo(a) & 70 & 19,9 \\
\hline Total & 351 & 100,0 \\
\hline
\end{tabular}




\begin{tabular}{|c|c|c|}
\hline \multicolumn{3}{|c|}{$\begin{array}{l}\text { QUADRO V. Consome ou consumiu nos últimos seis } \\
\text { meses algum «produto natural»/«suplemento } \\
\text { alimentar»? }\end{array}$} \\
\hline & $N$ & $\%$ \\
\hline Sim & 320 & 91,2 \\
\hline Não & 31 & 8,8 \\
\hline Total & 351 & 100,0 \\
\hline
\end{tabular}

\begin{tabular}{|c|c|c|c|}
\hline \multicolumn{4}{|c|}{$\begin{array}{l}\text { QUADRO VI. Caracterização demográfica dos } \\
\text { consumidores e não consumidores (Consome ou } \\
\text { consumiu nos últimos seis meses algum «produto } \\
\text { natural»/«suplemento alimentar»?) }\end{array}$} \\
\hline & & $\operatorname{Sim}(\%)$ & Não (\%) \\
\hline \multirow[t]{2}{*}{ Género } & Feminino & 62,4 & 6,6 \\
\hline & Masculino & 28,8 & 2,3 \\
\hline \multirow[t]{5}{*}{ Idade } & $18-30$ anos & 6 & 4 \\
\hline & $31-50$ anos & 23,9 & 3,1 \\
\hline & $51-65$ anos & 22,2 & 1,4 \\
\hline & $65-75$ anos & 22,2 & 0 \\
\hline & $>75$ anos & 16,8 & 0,3 \\
\hline \multirow[t]{5}{*}{ Escolaridade } & $\leq 4$ anos & 32,5 & 0,3 \\
\hline & 5-6 anos & 18,1 & 0,3 \\
\hline & 7-9 anos & 19 & 0,6 \\
\hline & Secundário & 12,9 & 2,3 \\
\hline & Superior & 9,2 & 4,9 \\
\hline \multirow[t]{4}{*}{ Estado civil } & Solteiro(a) & 8,8 & 4,6 \\
\hline & $\begin{array}{l}\text { Casado(a)/ } \\
\text { /União de facto }\end{array}$ & 55,3 & 3,7 \\
\hline & Divorciado(a) & 7,4 & 0,3 \\
\hline & Viúvo(a) & 19,7 & 0,3 \\
\hline
\end{tabular}

ram consumir os produtos em análise foram os utentes com menor nível de escolaridade $\left(\leq 4^{\text {a }}\right.$ classe: $32,5 \%)$. Em relação ao estado civil, 55,3\% dos utentes casados ou em união de facto confirmaram ter consumido os referidos produtos (Quadro VI).

Dos 91,2\% de utentes que responderam afirmativamente à questão anterior, a maioria referiu o consumo de um ou dois PN/SA (64,7\%). Apenas 11,2\% respondeu que tomava/tinha tomado quatro ou mais produtos/ suplementos (Quadro VII).

\begin{tabular}{|c|c|c|}
\hline \multicolumn{3}{|c|}{$\begin{array}{l}\text { QUADRO VII. Se sim, quantos «produtos»/ } \\
\text { /«suplementos» tomou? }\end{array}$} \\
\hline & $N$ & $\%$ \\
\hline 1 & 109 & 34,1 \\
\hline 2 & 98 & 30,6 \\
\hline 3 & 77 & 24,1 \\
\hline 4 & 18 & 5,6 \\
\hline 5 ou mais & 18 & 5,6 \\
\hline Total & 320 & 100,0 \\
\hline
\end{tabular}

\begin{tabular}{|c|c|c|}
\hline \multicolumn{3}{|c|}{$\begin{array}{l}\text { QUADRO VIII. Com que regularidade costuma tomar } \\
\text { esses produtos? }\end{array}$} \\
\hline & $N$ & $\%$ \\
\hline Todos os dias & 243 & 75,9 \\
\hline Em períodos limitados de tempo & 48 & 15,0 \\
\hline Raramente & 29 & 9,1 \\
\hline Total & 320 & 100,0 \\
\hline
\end{tabular}

O item seguinte do questionário incidiu sobre a regularidade na toma destes produtos, tendo-se constatado que $75,9 \%$ o fazia diariamente (Quadro VIII).

Analisando o motivo pelo qual os inquiridos consumiam os referidos produtos verificou-se que eram principalmente produtos para patologias do foro músculoesquelético $(17,1 \%)$ e para regularização do trânsito gastrointestinal (14,2\%) (Quadro IX).

Quando se questionou se o seu médico tinha conhecimento que tomava esses produtos/suplementos, $52,8 \%$ respondeu que não (Quadro X).

Para verificar a existência de associação entre o consumo de PN/SA e o género, a escolaridade e estado civil procedeu-se à realização do teste de Qui-Quadrado. Para verificar a existência de associação entre o consumo de PN/SA e a idade procedeu-se à realização do teste de correlação de Spearman (Quadro XI).

Através do Teste de Independência de Qui-Quadrado verificou-se que a variável «Género» e a questão Consome ou consumiu nos últimos seis meses algum produto natural/suplemento alimentar? não apresentam associação ( $p>0,05)$.

Avaliada a independência entre as variáveis «Idade», «Escolaridade» e «Estado Civil» e a questão Consome ou 


\begin{tabular}{|c|c|c|}
\hline \multicolumn{3}{|c|}{$\begin{array}{l}\text { QUADRO IX. Para que toma os «produtos } \\
\text { naturais»/«suplementos»? }\end{array}$} \\
\hline Questões & $N^{*}$ & $\%$ \\
\hline Ossos e articulações & 120 & $17,1 \%$ \\
\hline Cansaço, falta de energia e memória & 72 & $10,3 \%$ \\
\hline Emagrecimento & 8 & $1,1 \%$ \\
\hline Ansiedade, stress e insónia & 82 & $11,7 \%$ \\
\hline Prisão de ventre/obstipação & 100 & $14,2 \%$ \\
\hline Problemas gastrointestinais & 4 & $0,6 \%$ \\
\hline Impotência/Disfunção eréctil & 34 & $4,8 \%$ \\
\hline Menopausa & 24 & $3,4 \%$ \\
\hline Infeções urinárias & 37 & $5,3 \%$ \\
\hline Reforço da imunidade & 37 & $5,3 \%$ \\
\hline Prevenção cardiovascular & 2 & $0,3 \%$ \\
\hline Diminuição de açúcar no sangue & 14 & $2,0 \%$ \\
\hline Diminuição de colesterol no sangue & 41 & $5,8 \%$ \\
\hline Fortalecimento capilar e unhas & 44 & $6,3 \%$ \\
\hline Suplementos vitamínicos e minerais & 74 & $10,5 \%$ \\
\hline Suplementos adequados a desporto & 6 & $0,9 \%$ \\
\hline Outros & 3 & $0,4 \%$ \\
\hline Total & $702 *$ & $100,0 \%$ \\
\hline
\end{tabular}

*Valores referentes às escolhas múltiplas (daí o valor ser superior a 320).

\begin{tabular}{|c|c|c|}
\hline \multicolumn{3}{|c|}{$\begin{array}{l}\text { QUADRO X. O seu médico sabe que consome os } \\
\text { produtos que assinalou atrás? }\end{array}$} \\
\hline & $N$ & $\%$ \\
\hline Sim & 147 & 45,9 \\
\hline Não & 169 & 52,8 \\
\hline Total & 316 & 98,7 \\
\hline NS/NR & 4 & 1,3 \\
\hline Total & 320 & 100,0 \\
\hline
\end{tabular}

\section{DISCUSSÃO}

O presente estudo avaliou a prevalência do consumo de PN/SA nos utentes adultos inscritos na USF Arruda e a sua eventual associação com variáveis sociodemográficas (género, idade, escolaridade e estado civil).

No período de tempo em que decorreu esta investigação foram obtidos 351 questionários válidos.

Tratando-se de uma amostra de conveniência, não aleatória, as autoras do estudo consideram a possibilidade de existência de viés de seleção.

O uso de um questionário previamente validado por aplicação de um pré-teste apoia a fiabilidade dos resultados obtidos e demonstra a sua aplicabilidade.

De acordo com os resultados obtidos, $91,2 \%$ dos utentes consume ou consumiu nos últimos seis meses algum PN/SA, o que é um valor superior ao referido pelos estudos nacionais existentes até ao momento. Num estudo nacional publicado em 2006, com o objetivo de estudar os hábitos de consumo de PN/SA da população portuguesa, os resultados apontaram para um consumo de $81 \%$ (em que $38 \%$ se referiam a chás/infusões/ /plantas). Outro estudo nacional em 2008, que consumiu nos últimos seis meses algum produto natu$\mathrm{ral} /$ suplemento alimentar? verificou-se que as mesmas estão associadas $(p<0,05)$. estudou a população da região de Lisboa eVale do Tejo, mostrou que $48,8 \%$ dos inquiridos consumiram estes produtos. $^{1,7}$ 
Relativamente aos $91,2 \%$ de consumidores de PN/SA na presente investigação, a maioria referiu o consumo de um ou dois PN/SA $(64,7 \%)$ e $75,9 \%$ tinha um consumo diário. Os PN/SA mais consumidos eram os destinados aos ossos e articulações (17,1\%), à prisão de ventre/obstipação (14,2\%), à ansiedade, stress e insónia $(11,7 \%)$, os suplementos vitamínicos e minerais $(10,5 \%)$ e os produtos para alívio do cansaço, falta de energia e memória (10,3\%).

De ressalvar ainda que a maioria dos consumidores destes produtos $(52,8 \%)$ referiu que o seu médico não tinha conhecimento que tomavam esses PN/SA, valor inferior a um estudo publicado em 2014, onde 73,9\% dos consumidores de PN referia que o seu médico de família desconhecia que tinham este tipo de consumos. ${ }^{1}$

Relativamente à análise de associação entre o consumo de PN/SA e as variáveis demográficas estudadas (género, idade, escolaridade e estado civil) concluiu-se que apenas não há associação para a variável «Género». Deste modo, apesar de na presente amostra a maior percentagem de consumidores ser do género feminino, não se verificou associação estatisticamente significativa entre o consumo de PN/SA e o género.

Relativamente às restantes variáveis existe associação estatisticamente significativa com o consumo destes produtos. Verificou-se maior consumo nas faixas etárias dos 31-50 anos, 51-65 anos e 65-75 anos. No que diz respeito à escolaridade, o maior consumo ocorre nos utentes com menor grau de escolaridade ( $\leq 4$ anos). Analisando a relação do consumo destes produtos com o estado civil conclui-se que a maioria dos consumidores é casada ou vive em união de facto (Quadro VI).

Analisando os dados obtidos, provavelmente utentes com menor nível de escolaridade tendem a ser mais suscetíveis às estratégias de marketing destes produtos. Por outro lado, os resultados parecem apoiar que os casados ou a viver em união de facto sejam economicamente mais favorecidos e por esse motivo consumam mais este tipo de produtos.

Os resultados deste estudo apontam para uma elevada prevalência de consumo de PN/SA na USF Arruda. No entanto, atendendo ao número de utentes pertencentes à referida Unidade, para que a amostra fosse representativa da população em estudo, seriam necessários 366 questionários válidos, considerando um intervalo de confiança de 95\% e um erro de amostragem de $5 \%$. Neste caso, embora o número de questionários válidos (351) tenha um valor muito aproximado não permite que a presente amostra seja representativa da população em estudo.

Atendendo a que a presente amostra é constituída maioritariamente por indivíduos do sexo feminino $(68,9 \%)$ e de grupos etários mais avançados $(62,9 \%$ de inquiridos acima de 50 anos), aproxima-se da população habitual que recorre aos centros de saúde nacionais, ${ }^{8}$ o que, em conjugação com o facto de se ter obtido um número relativamente elevado de questionários válidos, leva a acreditar que estes resultados se possam aproximar do panorama nacional.

No entanto, para que os dados pudessem ser extrapolados para a totalidade da população portuguesa, seriam necessários estudos mais robustos, com amostras aleatórias e multicêntricas.

Atendendo a que este trabalho incide sobre uma temática atual e de interesse comum, associado ao facto de que existem poucos estudos nesta área e de que os escassos estudos existentes são pouco atuais, considera-se que seria pertinente realizar mais projetos de investigação com um maior número de inquéritos válidos, idealmente que pudessem ser representativos da realidade nacional.

Paralelamente considera-se importante a realização de estudos para determinar as razões de muitos médicos desconhecerem o consumo dos produtos em questão por parte dos seus utentes. Um dos benefícios na realização do presente estudo foi o de alertar os médicos para a importância de questionar os utentes acerca desta temática com vista à minimização das eventuais interações e reações adversas.

\section{REFERÊNCIAS BIBLIOGRÁFICAS}

1. Soares AL, Moutinho A, Velho D, Campos R, Teixeira A. Estudo Prev-Natura: estudo da prevalência do consumo de produtos naturais. Rev ADSO. 2014;2(3):36-44.

2. INFARMED. Produtos-fronteira entre suplementos alimentares e medicamentos [Internet]. Lisboa: INFARMED; [s.d.]. Available from: http://www.infarmed.pt/documents/15786/17838/PRODUTOS+FRO NTEIRA+SULEMENTOS+MEDICAMENTOS.pdf/d0cd8eOf-fad8-474b85b4-b32c01fac5e9

3. Decreto-Lei n 136/2003, de 28 de junho. Diário da República. I Série$A(147)$.

4. Parlamento Europeu, Conselho Europeu. Diretiva 2002/46/CE, de 10 de junho de 2002, relativa à aproximação das legislações dos Estados- 
Membros respeitantes aos suplementos alimentares. J Ofic Comun Europeias. 2002;L(183):51-7.

5. Mousinho C, Hergy F. Suplementos alimentares: o que são e como notificar reações adversas? Bol Farmocovigilância. 2017;21(3):1-2.

6. Barros L, Coimbra P, Matias C, Fernandes N, Rocha F, Órfão C, et al. Produtos naturais e avaliação pré-operatória. Rev Soc Port Anestesiol. 2007;16(4).

7. Santos AC, Oliveira S, Águas S, Monteiro C, Palma ML, Martins AP, et al. Recolha de dados sobre consumo de medicamentos e/ou suplementos à base de plantas medicinais numa amostra da população de Lisboa e Vale do Tejo [Data collection on the consumption of drugs and / or supplements based on medicinal plants in a population sample from Lisboa and Vale do Tejo]. Rev Lusóf Ciênc Tecnol Saúde. 2008;5(2):12841. Portuguese

8. Departamento de Consolidação e Controlo de Gestão do SNS. Esta- tística do movimento assistencial: centros de saúde, 2003 [Internet]. Lisboa: Instituto de Gestão Informática e Financeira da Saúde; 2003. Available from: http://www2.acss.min-saude.pt/Portals/0/DownloadsPublicacoes/SNS/Info_Activid/Estat_2003_CentSaude.pdf

\section{CONFLITOS DE INTERESSE}

Os autores declaram não possuir quaisquer conflitos de interesse.

\author{
ENDEREÇO PARA CORRESPONDÊNCIA \\ Ângela Lee Chin \\ E-mail: angelalee.chin@gmail.com \\ http://orcid.org/0000-0003-4313-2815
}

Recebido em 02-09-2017

Aceite para publicação em 21-11-2018

\section{ABSTRACT}

\section{CONSUMPTION OF «NATURAL PRODUCTS» AND «DIETARY SUPPLEMENTS» IN A FAMILY HEALTHCARE UNIT: AN EXPLORATORY STUDY}

Introduction: The consumption of «natural products» (NP) and «dietary supplements» (DS) has increased in popularity. Studies on the consumption of these products are important because there are few national data, and the population is usually unware of the associated risks.

Objectives: To determine the prevalence of NP/DS consumption in the Arruda Family Healthcare Unit. Additionally, it was aimed to socio-demographically characterise the consumers of these products, their consumption habits, and to verify if there is an association with gender, age, schooling and marital status.

Type of study: Observational, cross-sectional and descriptive.

Setting: Arruda Family Healthcare Unit.

Population: Adult users enrolled in Arruda Family Healthcare Unit.

Methods: Data were collected using a non-randomised convenience sample, between October 1 and November 30, 2016. An anonymous, confidential and voluntary questionnaire was applied. Data were coded, recorded and analyzed in SPSS ${ }^{\circledR}$.

Results: There were 351 valid questionnaires. Amongst the respondents, $68.9 \%$ were women, $72.9 \%$ were aged between 31 and 75 years old, $50.7 \%$ had six or less years of schooling, and $50.9 \%$ were married or lived as a couple. $91.2 \%$ reported consuming or having consumed NP/DS in the last six months. Of these, $64.7 \%$ consumed one or two NP/DS, and $75.9 \%$ did so daily. Analysing the association between NP/DS consumption and the studied variables, it was concluded that were association with all variables but gender.

Conclusions: The results of this study point to a high prevalence of NP/DS consumption in Arruda Family Healthcare Unit. Given the relevance of this issue and the fact that there are few current studies in this area, we consider that it would be pertinent to carry out more robust investigations, ideally that could be representative of the national landscape.

Keywords: Natural products; Dietary supplementations; Family physician 